Revista lus et Praxis, Año 27, № 1, 2021, pp. 143 - 156

ISSN 0717 - 2877

Universidad de Talca - Facultad de Ciencias Jurídicas y Sociales

La pre-action disclosure y las diligencias preliminares:

un estudio comparado

Roser Casanova Martí

Fecha de recepción: 2019-09-03; fecha de aceptación: 2020-01-16

\title{
La pre-action disclosure y las diligencias preliminares: un estudio comparado ${ }^{1,2}$
}

THE PRE-ACTION DISCLOSURE AND THE PRELIMINARY PROCEEDINGS: A COMPARATIVE STUDY

ROSER CASANOVA MARTí ${ }^{3}$

\section{RESUMEN}

A veces, quizás más de lo deseado, las partes acuden a los tribunales de justicia sin contar con todas las herramientas necesarias para poder afrontar el proceso de manera eficaz. Siendo esto así, ¿̇es posible que las futuras partes puedan llevar a cabo alguna actuación previa para iniciar el proceso en mejores condiciones, o incluso llegar a evitarlo? Como es bien sabido, la respuesta es afirmativa en el caso español, que regula las diligencias preliminares, si bien de manera incompleta, en la Ley de Enjuiciamiento Civil (en adelante LEC) ${ }^{4}$. Con el objetivo de alcanzar el propósito inicialmente indicado, se hace necesario ahondar en el análisis de otras instituciones procesales afines existentes en el derecho comparado para intentar mejorar el funcionamiento de las diligencias preliminares. Una figura similar es la pre-action disclosure del ordenamiento jurídico inglés, que se encuentra regulada en las Civil Procedure Rules (en adelante CPR) y sus respectivas Practice Directions (en adelante PD) 5 .

El presente trabajo tiene como finalidad examinar las notas características de ambas instituciones procesales, así como sus puntos de encuentro y desencuentro a la luz de la doctrina judicial española e inglesa ${ }^{6}$.

\section{PALABRAS CLAVE}

Prueba, pre-action disclosure, diligencias preliminares

\section{ABSTRACT}

Sometimes, perhaps more than desired, parties go to court lacking of all the enabling tools needed in order to face the trial successfully. When this happens, would it be possible that future parties could be carry out some preliminary action to start the process under better conditions, or even avoid it? As is well known, the answer is affirmative in the Spanish case that, though incompletely, regulates the preliminary proceedings in the Civil Procedure Act. In order to achieve the initially indicated purpose, it is necessary to deep in the analysis of other related procedural institutions existing in comparative law to try to improve Spanish regulation. One of those institutions is the pre-action disclosure of the English legal system, as regulated in the Civil Procedure Rules and their respective Practice Directions.

The aim of this paper is to examine the characteristics of both procedural institutions as well as their points of encounter and disagreement bearing in mind Spanish and English judicial doctrine.

\footnotetext{
${ }^{1}$ Este trabajo es el resultado de una estancia de investigación realizada en la University of Oxford durante los meses de mayo a julio de 2019, auspiciada por el profesor Adrian Zuckerman, a quien agradecemos las facilidades bibliográficas concedidas.

2 Este trabajo se enmarca en el proyecto I+D “Hacia una nueva regulación de la prueba pericial” (DER2016-7549-P), financiado por el Ministerio de Economía y Competitividad de España, y el Grupo de Investigación Consolidado "Evidence Law" (2017 SGR 1205 ) financiado por la Agència de Gestió d'Ajuts Universitaris i de Recerca de la Generalitat de Catalunya (AGAUR), en ambos como investigador principal el profesor Joan Picó i Junoy.

3 Doctora Profesora Doctora de Derecho Procesal, Universitat Rovira i Virgili, Tarragona, España. Correo electrónico roser.casanova@urv.cat.

${ }^{4}$ Ley № 1, de 2000. Disponible en https://www.boe.es/buscar/act.php?id=BOE-A-2000-323.

${ }^{5}$ Disponible en http://www.justice.gov.uk/courts/procedure-rules/civil/rules/part31.

${ }^{6}$ De la colaboración de las partes en una fase temprana del procedimiento en los países del common law se ha preocupado también la doctrina procesal chilena, siendo de destacar: PEÑA (2017).
} 


\section{KEYWORDS}

Evidence, pre-action disclosure, preliminary proceedings

\section{El intercambio de documentos antes de la pre-action disclosure}

El ordenamiento jurídico británico establece el deber de cooperación entre partes consistente en el intercambio de información antes de iniciar un proceso judicial. Esto significa que, sin intervención judicial alguna, las partes, como regla general, se exhibirán recíprocamente cualquier documentación relevante y esencial. Esta revelación prejudicial de documentos, que caracteriza el proceso civil inglés, implica que los posibles litigantes puedan, y habitualmente lo hacen, establecer una lista de los documentos que deseen ver, existiendo la expectativa de que la parte reclamada efectivamente los revelará o, por contra, explicará los motivos por los cuales los documentos reclamados no deben mostrarse ${ }^{7}$.

Así pues, y para alcanzar este propósito, en cualquier controversia se espera que las partes cumplan con lo establecido en la Practice Direction Pre-Action Conduct and Protocols (en adelante PDPAC) ${ }^{8}$ de las CPR. Esta directriz práctica establece la conducta que las partes debieran tener antes de iniciar el proceso judicial, y que hace referencia al hecho de que haya existido un intercambio de información suficiente para: comprender la posición de la otra parte; decidir, en su caso, cómo proceder; evitar el inicio del proceso judicial; optar por resolver el conflicto a través de un ADR (Alternative Dispute Resolution); y/o reducir costes. No obstante, para determinadas reclamaciones civiles se prevén unos protocolos específicos, enumerados en el párrafo 18 de la propia PDPAC, aprobados por el Master of the Rolls ${ }^{9} \mathrm{y}$ anexados también a las CPR, lo que explica que en defecto de un protocolo específico deba estarse a la genérica previsión de la PDPAC.

Sin embargo, en ocasiones el requerido puede incumplir no mostrando todos los documentos a su alcance que le han sido solicitados. Solo entonces el requirente insatisfecho con la revelación de documentos o su contenido podrá solicitar al órgano judicial una pre-action disclosure en virtud de la rule $31.16 \mathrm{CPR}^{10}$ para que ordene tal revelación.

Asentadas las anteriores premisas, estamos en disposición de definir la pre-action disclosure como el mecanismo procesal que permite a las partes obtener respectivamente, a través de una orden judicial, información relevante y completa sobre el material documental de las otras, tan pronto como sea posible, con alguno de los siguientes propósitos: obtener la suficiente información para conocer los puntos fuertes del asunto; valorar la judicialización del conflicto y, por lo tanto, la conveniencia de seguir adelante con el litigio; e, incluso, evaluar la posible evitación del pleito ${ }^{11}$.

\footnotetext{
${ }^{7}$ Sobre esta cuestión véase también BLACKIE y BATES (2014), p. 230.

8 Ver https://www.justice.gov.uk/courts/procedure-rules/civil/rules/pd_pre-action_conduct. A mayor abundamiento, véase HOLLANDER (2015), pp. 1-5; y CUNNINGHAM-HILL y ELDER (2013), p. 318.

${ }^{9}$ El Master of the Rolls es un juez del Tribunal de Apelación y, a su vez, el Presidente de su División Civil, sobre quien recae la responsabilidad de organizar el trabajo de los jueces de su división, así como presidir uno de sus tribunales. Para más información véase https://www.judiciary.uk/about-the-judiciary/who-are-the-judiciary/judicial-roles/judges/profile-mor/.

${ }^{10}$ Véase CUNNINGHAM-HILL y ELDER (2013), p. 318.

${ }^{11} \mathrm{El}$ deber de cooperación entre las partes para obtener la revelación de documentos tradicionalmente solo se preveía una vez iniciado el proceso judicial, pero no antes. Para evitar las dificultades que pudieran tener las víctimas de lesiones para acceder a la documentación que pudiera determinar la prosperabilidad de una posterior acción judicial de reclamación para el resarcimiento de tales lesiones - por ejemplo, el acceso del paciente a su historial clínico-, el "Report of the Committe on Personal Injuries Litigation" recomendó introducir la "pre-action discovery" en las reclamaciones de lesiones personales, facultando al tribunal para ordenar la revelación de documentos previa a la acción, a petición de quien pudiera ser parte en un proceso posterior. Finalmente, la preaction disclosure se extendió a todas las reclamaciones, encontrando actualmente expreso reflejo en la regla 31.16 CPR. ZUCKERMAN (2013), pp. 756-757.
} 


\section{El deber de disclosure previo inicio del proceso judicial}

\section{1. Ámbito de aplicación}

El deber de disclosure se prevé, por regla general, en el seno de un proceso judicial (rule 31.1 (PR) y consiste en la obligación de las partes de revelar documentos que están o hayan estado en su poder y resulten de interés para el proceso con la finalidad que las otras partes puedan inspeccionar su contenido, manteniéndose este deber durante todo el proceso hasta su conclusión, de manera que si en el transcurso del proceso una parte tiene noticia de cualquier otro documento relevante para el juicio deberá notificarlo inmediatamente a las otras partes (rule $31.11 \mathrm{CPR})^{12}$.

Sin embargo, excepcionalmente se permite que una de las partes solicite al tribunal, antes de iniciar el proceso, la revelación de documentos a otra persona que en este momento ni tan siquiera ostenta la condición de parte, conforme a la regla $31.16 \mathrm{CPR}^{13}$. Esta posibilidad que nos ofrece el ordenamiento jurídico inglés no se aplicará cuando se opte por la vía arbitral para resolver la relación jurídica subyacente ${ }^{14}$.

\subsection{Requisitos para la adopción de una pre-action disclosure}

El deber de disclosure en un momento anterior al proceso judicial, como no podía ser de otra manera, se solicitará ante el órgano judicial quien, posteriormente, deberá autorizarlo.

Dicha autorización judicial puede, que no debe, acordar la disclosure cuando concurran los siguientes tres presupuestos, que serán desarrollados en los epígrafes que siguen: en primer lugar, la posibilidad que solicitante (applicant) y solicitado (respondent) sean partes del posterior proceso judicial; en segundo lugar, que la revelación se extienda a los documentos o clases de documentos propios de la standard disclosure; $y$, por último, que concurran los requisitos para que el órgano judicial pueda valorar la viabilidad de la pre-action disclosure en el concreto caso.

No obstante, debe tenerse en cuenta que el simple cumplimento de los anteriores requisitos no comporta automáticamente la emisión de una resolución judicial que acuerde el deber de revelación, y ello se ha encargado de explicarlo la doctrina judicial ${ }^{15}$. Así, el tribunal, previa resolución, deberá también valorar la probabilidad de que la pretensión pueda someterse a juicio y que los documentos solicitados para su revelación sirvan efectivamente para respaldar cualquiera de las dos futuras posiciones procesales. Solo entonces el órgano judicial estará en condición de decidir si aprueba la revelación de documentos.

\subsubsection{The respondent and the applicant are likely to be parties to subsequent proceedings (requirente y requerido deben encontrarse en posición de ser, eventualmente, litigantes)}

La primera cuestión que debe considerar el tribunal para acordar la revelación de documentos antes de iniciar el proceso judicial, y siguiendo el tenor literal de la ley, es que sea posible que el requerido (respondent) y el requiriente (applicant) sean partes del futuro proceso judicial. En este punto debe matizarse que el término likely que utiliza la norma no debe confundirse con el de probable entendido este segundo como una más alta probabilidad de que sean partes, sino que debe asimilarse a la expresión may well (quizás). De hecho, así lo han

12 Confróntese CERRATO (2015), pp. 309-333.

13 ILLER (2006), pp. 330-331, reconoce que a diferencia de lo que sucedía antes de la entrada en vigor de las CPR, la posible exigencia de revelación de documentos previo inicio del proceso se ha extendido a cualquier tipo de proceso, más allá de las reclamaciones por lesiones personales o accidentes mortales.

${ }^{14}$ En esta misma línea, SMITH (2015), p. 845; y, ASHFIELD et al. (2015), p. 915, este último con cita al caso EDO Corporation vs. Ultraelectronics Ltd [2009] EWHC 682 (Ch), [2009] Bus LR 1306.

${ }^{15}$ En este sentido véase DI MAMBRO (2019), p. 882, sobre la base del caso Bermuda International Securities Ltd vs KPMG (a Firm) [2001] EWCA Civ 269, (2001) 145 Sol Jo LB 70. Respecto de la capacidad de decisión del juez, más allá de los requisitos del art. 31.16 CPR, véase SMITH (2015), p. 845. 
considerado los tribunales ingleses quienes han clarificado esta compleja cuestión planteándose si el sentido de la norma requiere que sea probable la posterior iniciación del proceso judicial, o bien tan solo que las personas involucradas puedan llegar a ser parte en caso de que tuviere lugar un proceso judicial ${ }^{16}$, siendo suficiente con que exista alguna posibilidad (may well), por remota que sea ${ }^{17}$.

\subsubsection{Documents or classes of documents of the standard disclosure (documentos o tipos de documentos de la revelación estándar)}

La segunda exigencia cuyo cumplimento debe valorar el tribunal es si los documentos objeto de revelación se encuentran entre los relacionados en la regla 31.6 CPR prevista para la standard disclosure. En concreto son los que pudieran dar lugar a alguna de las tres siguientes casuísticas: los documentos sobre los que pueda sostenerse la pretensión; aquellos otros que pudieran perjudicar a la propia parte que los aportase o bien a la contraparte; $y$, por último, la exhaustividad de este precepto legal extiende el deber de disclosure incluso a los documentos que pudieran beneficiar a la otra parte ${ }^{18}$. Por lo tanto, una interpretación negativa de esta norma nos lleva a la conclusión de que la pre-action disclosure no podrá tener por finalidad aquellos documentos que tampoco pudieran ser objeto de revelación en caso de que se hubiera iniciado el proceso judicial ${ }^{19}$.

\subsubsection{Disclosure before proceedings have started should be desirable (es deseable que la disclosure haya comenzado antes de iniciar los procedimientos judiciales)}

Finalmente, será imprescindible que concurra alguno de los requisitos del apartado d) de la rule 31.16 (3) CPR para que el órgano judicial pueda valorar si en el concreto caso la pre-action disclosure debe tener lugar, a saber:

a. poner a disposición del futuro proceso todos los documentos para alcanzar un resultado justo, de acuerdo con las reglas del fair play,

b. llegar a un acuerdo que evite el futuro proceso, y/o

c. ahorrar los costes del futuro proceso judicial.

Por ello, teniendo en cuenta que el deber de revelación documental rige generalmente una vez iniciado el procedimiento judicial, siendo poco frecuente que suceda antes de la interposición de la demanda, el tribunal deberá valorar con la máxima exhaustividad posible si realmente la orden judicial de pre-action disclosure es necesaria (desirable).

Adicionalmente, y aunque la norma procesal inglesa no lo haya especificado, el tribunal deberá asimismo tener en consideración todas las circunstancias suscitadas en torno al caso concreto, y que se han encargado de matizar la doctrina judicial y científica. Siguiendo a Matthews y Malek $^{20}$, estas circunstancias son, principalmente, las que a continuación se enumeran:

- Si las partes han cumplido con el pre-action protocol o el pre-action enquires ${ }^{21}$.

- Si las partes han actuado diligentemente antes de acudir a la pre-action disclosure. En caso afirmativo, el tribunal no debería dictarla pues se entiende que el reclamado ha

\footnotetext{
${ }^{16}$ ZUCKERMAN (2013), p. 760

${ }^{17}$ Véase CUNNINGHAM-HILL y ELDER (2013), p. 318; y, ASHFIELD et al. (2015), p. 915, con cita al caso Herbert Black and Others vs. Sumitomo Corp [2002] 1 WLR 1562, [71].

${ }^{18}$ La falta de encaje en alguno de los supuestos anteriores tendrá como consecuencia el rechazo del tribunal de la solicitud de revelación de documentos. Precisamente esto es lo que sucede en el caso Pineway Ltd. vs. London Mining Co Ltd [2010] EWHC 1143 (Comm), respecto del cual SMITH (2015), p. 845, destacan que "an application was refused where it was unclear that the documents would fall within standard disclosure, and it appeared to be 'fishing'".

${ }^{19}$ De igual modo véase ASHFIELD et al. (2015), p. 915.

${ }^{20}$ MATTHEWS y MALEK (2017), pp. 93-96.

${ }^{21}$ Briggs \& Forrester Electrical Ltd vs. Governors of Southfield School [2005] EWHC 1734 (TCC) at [18], [19], [47]; y Marshall (Executors of Marshall) vs. Alliotts (A Firm) [2004] EWHC 1964 (QB), [2005].
} 
accedido voluntariamente a las peticiones del reclamante. En cambio, de no ser así, el tribunal podría adoptar una orden judicial de pre-action disclosure ${ }^{22}$.

- Los documentos que están en manos del applicant y los que han sido facilitados voluntariamente por el respondent.

- La naturaleza de la reclamación ${ }^{23}$.

- La sostenibilidad y fundamentación de la reclamación. A este respecto, deberá el tribunal examinar si la reclamación es fundamentada, en cuyo caso es posible que adopte la orden, en cambio, si se trata de una pretensión especulativa y poco justificada serán pocas las posibilidades que tendrá el applicant de que el tribunal acepte la preaction disclosure ${ }^{24}$. Sin embargo, no debe olvidarse que, incluso en los supuestos en los que la pretensión sea especulativa o de débil fundamentación, el tribunal discrecionalmente podrá decidir emitir la orden ${ }^{25}$.

- La naturaleza e importancia de los documentos requeridos. Debe tenerse en cuenta que si los documentos no están directamente relacionados con la reclamación (peripheral) existe el riesgo de que el tribunal rechace la solicitud ${ }^{26}$.

- Las opciones que tiene el applicant de iniciar el procedimiento judicial sin la necesidad de pedir la pre-action disclosure. Una vez más, tendrá la última palabra el órgano judicial, quien discrecionalmente decidirá sobre su adopción ${ }^{27}$.

- La posibilidad de obtener los documentos requeridos para su revelación prejudicial por otras vías diferentes, a no ser que tales vías fueran inadecuadas o implicasen a las partes costes extraordinarios ${ }^{28}$.

- La claridad y concreción de la reclamación, así como la delimitación de la revelación pretendida. Ello favorecerá una orden judicial dictada sobre la base de la transparencia en cumplimiento de los intereses de la justicia y la proporcionalidad debida ${ }^{29}$.

- El análisis individualizado, por parte del tribunal, de cada uno de los documentos y clases de documentos solicitados para su revelación ${ }^{30}$.

\subsection{Procedimiento}

Como hemos comentado anteriormente, la solicitud de revelación documental antes de iniciar el proceso judicial precisa del cumplimiento previo de cualquier pre-action protocol. Solo entonces el requirente insatisfecho podrá instar una pre-action disclosure ante el órgano judicial a través de una solicitud que se presentará de acuerdo con el formulario N244 (application notice in form N244) ${ }^{31,32}$. En esta solicitud, que deberá ir acompañada de prueba escrita (written evidence) -siguiendo la literalidad del apartado segundo de la rule 31.16 CPR-, el solicitante

\footnotetext{
${ }^{22}$ Black vs. Sumitomo Corporation [2001] EWCA Civ 1819, [2002] at [85]; y Gas Marketing Ltd vs. Centrica Storages Ltd [2009] EWHC 732 (Ch) at [27].

${ }^{23}$ Black vs. Sumitomo Corporation [2001] EWCA Civ 1819, [2002] at [83]; y Jet Airways (India) vs Barloworld Handling Ltd [2014] EWCA Civ 1311.

${ }^{24}$ Snowstar Shipping Co Ltd vs. Graig Shipping Plc [2003] EWHC 1367 (Comm) at [33]; BSW Ltd vs. Balltec Ltd [2006] EWHC 822 (Ch); Gwelhayl Ltd vs. Midas Construction Ltd [2008] EWHC 2316 (TCC); y Kneale vs. Barclays Bank (t/a Barklaycard) [2010] EWHC 1900 (Comm).

${ }^{25}$ En el caso Jay vs. Wilder Coe [2003] EWHC 1786 (QB) Tugenhart H. el tribunal manifiesta que la "pre-action disclosure could be appropiate even where the complain may be speculative or application arguably constitued a 'fishing expedition'"; $y$, en la misma línea, véase el caso Arsenal Football Club vs. Elite Sports Distribution [2002] EWHC 3057 (Ch), Lightman J.

${ }^{26}$ Northumbrian Water Ltd vs. British Telecommunications Plc [2005] EWHC 2408 (TCC) at [32].

${ }^{27}$ En el caso XL London Market Ltd vs. Zenith Syndicate Management Ltd [2004] EWHC 1182 (Comm) at [24] se indica que "where it was observed that it is a powerful argument against an order that the applicant can well make a case without the disclosure sought". Asimismo se manifiesta el caso First Goulf Bank vs. Wachovia Bank National Association [2005] EWHC 2827 (Comm).

${ }^{28}$ Hays Specialist Recruitment (Holdings) Ltd vs. Ions [2008] EWHC 745 (Ch) at [49], [50].

${ }^{29}$ Moresfield vs. Banners [2003] EWHC 1602 (Ch) at [32]; y Snowstar Shipping Co Ltd vs. Graig Shipping Plc [2003] EWHC 1367 (Comm) at [35].

${ }^{30}$ Hands vs. Morrison Construction Services Ltd [2006] EWHC 2018 (Ch) at [29], [70].

31 Ver: https://assets.publishing.service.gov.uk/government/uploads/system/uploads/attachment_data/file/732360/N244_web_0818.pd f.

${ }^{32}$ Así lo indica ASHFIELD et al. (2015), p. 917.
} 
indicará la naturaleza de sus alegaciones y explicará qué aporta cada uno de los documentos solicitados para la futura reclamación ${ }^{33}$.

La resolución judicial que acuerde el deber de revelación documental se dictará de conformidad con el contenido que establecen los apartados 4ㅇ y 5o de la rule 31.16 CPR, de carácter imperativo y potestativo, respectivamente.

Así, en primer lugar, obligatoriamente especificará los documentos que el solicitado (respondent) debe revelar por estar en su poder (possession, custody or power) y ser relevantes para el caso ${ }^{34}$. Además, el legislador ha previsto también los supuestos en los que tal revelación no pueda llevarse a término porque el documento ya no está en posesión del respondent o bien porque existe una causa que impide su revelación. En particular, el referido apartado 4우 requiere que se hagan constar, por un lado, los documentos que, aun estando sujetos a revelación, ya no se encuentran en poder del solicitado, en cuyo caso, el tribunal podrá exigirle además que justifique los motivos por los cuales ha dejado de disponer de dichos documentos (rule 31.16 (5) (a) CPR). Y, por el otro, aquellos documentos que no podrán revelarse por riesgo de dañar el interés público, al amparo de la rule 31.19 en relación con la PD 31A.6 CPR ${ }^{35}$.

En segundo lugar, el tribunal tendrá la facultad de incluir en la resolución judicial la hora y el lugar concretos para llevar a cabo la revelación por parte del respondent y la inspección de los documentos por el applicant.

Una cuestión controvertida que puede plantearse es la negativa del respondent de atender la pre-action disclosure. Al respecto, la regla 31.21 CPR ha previsto las consecuencias del incumplimiento de este deber vetando, a quien no quiso revelarlos, la posibilidad de utilizar los documentos durante el proceso judicial, excepto que el órgano judicial lo permita ${ }^{36}$.

Para finalizar debe en este punto igualmente considerarse el hecho que, aunque sea el reclamante (applicant) quien habitualmente realice la solicitud de pre-action disclosure, ello no siempre es así. Puede suceder que, tras haber sido requerido para revelar documentos, por su parte, el reclamado (respondent) se vea también en la necesidad de presentar esta solicitud de revelación documental respecto de algún documento que pueda estar o hubiere estado en posesión del applicant. En cualquier caso, sea quien fuere el sujeto solicitante, ¿quién afronta sus costes? La respuesta la encontramos en las reglas sobre costes de la parte 46 CPR. La regla general establece que sea la persona contra la que se dirige la orden judicial de pre-action disclosure (respondent) quien asuma los costes por esta generados. En nuestra opinión, es razonable que así sea pues podría haberse evitado este trámite procesal si el reclamado hubiere atendido al pre-action protocol. Aun así, teniendo en cuenta las circunstancias del caso - por ejemplo, la oposición del respondent a la solicitud, o el cumplimiento del pre-action protocol-, el tribunal podrá discrecionalmente condenar en costas a ambas partes o, incluso, al applicant cuando la solicitud sea injustificada ${ }^{37}$.

\subsection{Rechazo de la pre-action disclosure}

Del contenido de la norma que regula esta institución procesal nada se desprende de los motivos que pueden llevar al órgano judicial a rechazar la solicitud de pre-action disclosure. Por ello, una vez más, será necesario complementar la regulación de las CPR con la doctrina judicial y científica, quien sí se ha pronunciado sobre esta cuestión destacando principalmente las dos siguientes realidades.

\footnotetext{
${ }_{33}$ MATTHEWS y MALEK (2017), pp. 96-97.

${ }^{34}$ MATTHEWS y MALEK (2017), p. 97.

${ }^{35}$ Además, entendemos que pueden también excepcionarse del deber de disclosure aquellos casos en los que el tribunal lo considere oportuno (rule 31.5 (1) (b) CPR); o cuando las propias partes pacten por escrito esta dispensa (rule 31.5. (1) (c) CPR). Confróntese CERRATO (2015), p. 311.

${ }^{36}$ En esta línea se pronuncia DE LA PRADA y MUÑOZ (2014), p. 115, quienes añaden que "de querer servirse de ellos deberán cumplir el referido deber, salvo que cuenten con una autorización específica del tribunal".

${ }^{37}$ A mayor abundamiento, ASHFIELD et al. (2015), pp. 917-918; y, MATTHEWS y MALEK (2017), p. 98.
} 
De un lado, el riesgo que tiene el solicitante de la pre-action disclosure es que si el órgano judicial considera que la pretensión no tiene fundamento ello puede conducirle a la inadmisión de la futura demanda ${ }^{38}$. Por lo que antes de solicitar la pre-action disclosure el solicitante debe valorar la sostenibilidad de su pretensión pues corre el peligro de ver frustrada su demanda incluso antes de presentarla.

Así pues, una vez presentada la solicitud corresponderá al órgano judicial determinar la viabilidad del asunto y, en consecuencia, su posibilidad real de éxito. Aunque este no es el momento procesal de entrar a valorar sobre el fondo del asunto, inevitablemente el tribunal deberá considerar si la futura demanda es sostenible. Siendo esto así, solo en los casos en los que la pretensión carezca de los mínimos exigibles para su prosperabilidad (hopeless or nonjusticiable) el tribunal inadmitirá el deber de revelación documental solicitado en virtud de la pre-action disclosure ${ }^{39}$. Ello sucedería, por ejemplo, en el caso de que el tribunal tuviese dudas sobre si los documentos, cuya revelación se pretendiera, pudieran realmente hacer prueba para sostener la futura reclamación al considerarla especulativa ${ }^{40}$. En cambio, el tribunal no debería rechazar de plano la disclosure en esta fase preliminar del procedimiento cuando el objeto de la controversia fuese complejo o careciera de suficientes elementos para evaluar la solidez del caso $^{41}$.

Y, de otro lado, el solicitante debe también valorar, previa presentación de la solicitud, si realmente es necesaria tal revelación documental o bien si es posible llevar a cabo la futura acción judicial prescindiendo de ella cuando ya tuviere suficiente material para presentar su demanda -aunque no tan completa como si se hubiera realizado dicha pre-action disclosurepues de no ser así, si el órgano judicial finalmente entiende que la revelación era innecesaria, podrá rechazarla ${ }^{42}$.

\section{Las diligencias preliminares}

Una vez hemos analizado el funcionamiento de la pre-action disclosure, a continuación, pretendemos realizar un estudio comparativo de esta institución característica del common law en relación con las diligencias preliminares españolas, por la afinidad habida entre ambas instituciones procesales ${ }^{43}$. En el ordenamiento jurídico español las diligencias preliminares se encuentran defectuosamente reguladas en los arts. 256 y siguientes de la LEC ${ }^{44}$, motivo por el cual el presente apartado se desarrolla esencialmente sobre la base de la interpretación que de las mismas ha realizado la doctrina judicial. En particular, nos centramos en aquellas cuestiones de las diligencias preliminares que presentan algún punto de conexión con la ya examinada pre-

\footnotetext{
${ }^{38}$ El caso Smith vs. Secretary of State for the Environment and Climate Change [2013] EWCA Civ 1585, [2014] 1 WLR 2283, Underhill $\sqcup$, pone de manifiesto la discrecionalidad del órgano judicial a la hora de tomar esta decisión al considerar que: “...it is inherently better that questions about the likelihood of the applicant being able in due course to establish a viable claim are considered as part of a flexible exercice of the court's discretion in the context of the particular case". Confróntese DI MAMBRO (2019), p. 882.

${ }^{39}$ En este sentido se pronuncia la doctrina judicial en el caso Smith vs. Secretary of State for the Envirorment and Climate Change [2013] EWCA Civ 1585, [2014] 1 WLR 2283, Underhill LJ.

${ }^{40}$ Confróntese caso Herbert Black and Others vs. Sumitomo Corp [2002] 1 WLR 1562, [71].

${ }^{41}$ AAVV., Blackstone's Civil Practice..., ob. cit., pp. 916-917, con cita al caso Anglo Irish Bank Corporation plc vs. West LB AG [2009] EWHC 207 (Comm), LTL 25/2/2009.

${ }^{42}$ Streamship Mutual Underwriting Association Trustees (Bermuda) Ltd vs. Baring Asset Management Ltd [2004] EWHC 202 (Comm), [2004] All ER (D) 272 (Feb), Aikens J. Confróntese DI MAMBRO (2019), p. 882. Siguiendo a ZUCKERMAN (2013), p. 761, este abuso podría llevar a la consideración de antieconómica la pre-action disclosure, de modo que esta institución procesal no debería adoptarse simplemente para que el solicitante pueda realizar un mejor enfoque de su posterior reclamación judicial.

${ }^{43}$ La equivalencia de esta institución procesal en el ordenamiento jurídico chileno son las medidas prejudiciales reguladas en los arts. 273 (263) a 302 (292) del Código de Procedimiento Civil (Ley 1552, de 1902). Disponible en https://www.leychile.cl/Navegar?idNorma=22740.

${ }^{44}$ Especialmente crítica con la anterior y vigente regulación de las diligencias preliminares se manifiesta CASTRILLO (2018), p. 407 quien, aun así, considera que estamos ante una institución útil en la práctica mostrando por ello el "convencimiento de que su estudio detallado contribuirá a clarificar su regulación y, por tanto, a allanar el terreno de quienes se planteen hacer uso de las diligencias preliminares" (p. 27). Y de hecho así lo hace a través de su brillante monografía que analiza en profundidad todas las cuestiones relativas a las diligencias preliminares, a la que, para un más amplio estudio de esta materia, nos remitimos. Igualmente, crítica en este punto es la recentísima aportación de RICHARD (2019), pp. 1-12. A mayor abundamiento, nos remitimos también a CORBAL (2014), pp. 3483-3602.
} 
action disclosure, dejando al margen las peculiaridades que sean propias de la institución española por exceder del objeto de la presente investigación.

\subsection{Concepto y finalidad}

Las diligencias preliminares son un instrumento procesal a disposición de quien pretenda obtener información relevante antes de iniciar un proceso judicial y que tienen por finalidad solicitar al órgano judicial - un juez de primera instancia o de lo mercantil, según proceda (art. 257 LEC) - la práctica de determinadas actuaciones con el objetivo de preparar adecuadamente el futuro proceso judicial o, incluso, y aunque la norma procesal no lo haya previsto expresamente, evitarlo ${ }^{45}$.

Ahondando en la primera de las finalidades indicadas, la Audiencia Provincial de Barcelona, en su reciente auto de 20 de septiembre de 2018 ha indicado que "[...] Las diligencias preliminares tienen la finalidad de preparar un posterior juicio declarativo, recabando la información necesaria para decidir sobre la procedencia de su interposición y el alcance de las pretensiones a ejercitar, completando aquellos datos que se ignoran acerca de la personalidad, la legitimación de la parte contraria o el objeto del procedimiento"46. Se trata, en definitiva, de obtener toda la información indispensable para que, en caso de que acabe iniciándose, el futuro proceso judicial sea eficaz ${ }^{47}$. Y en relación con el propósito de evitar el posterior proceso judicial, en palabras de la Audiencia Provincial de Zamora, en su auto de 8 de enero de 2003, "no tienen estas diligencias que desembocar necesariamente en un proceso sino que es perfectamente legítimo el que, tras ese examen de los elementos documentales o documentadores solicitados, se opte por no ejercitar acción alguna" 48 .

A diferencia de lo que sucede en la normativa española, la inglesa regula de forma más detallada las finalidades pretendidas por la pre-action disclosure. De manera específica, en primer lugar, y coincidiendo en parte con el propósito del art. 256 LEC, el legislador inglés prevé poner a disposición del futuro proceso todos los documentos para alcanzar un resultado justo, que en su caso servirá para la preparación del posterior proceso judicial. Pero no se limita solo a esta finalidad, sino que expresamente amplía su ámbito de aplicación a la evitación del futuro proceso - aunque esta finalidad no se encuentra explícitamente prevista en la regulación española sí ha sido reconocida a nivel jurisprudencial-. Y, por último, también se extiende a la posibilidad de ahorrar los costes del futuro proceso judicial, lo que ni tan siquiera ha sido recogido, en estos términos, en el sistema procesal español.

\subsection{Relación tasada de las diligencias preliminares}

Una de las cuestiones controvertidas en torno a las diligencias preliminares y que no se desprende del tenor literal de la ley es si se encuentran sujetas a un numerus clausus o bien son ilimitadas las actuaciones que pueden llevarse a cabo en este momento prejudicial. Esta falta de concreción se ha trasladado al ámbito de la doctrina judicial donde mayoritariamente se ha apreciado que solo pueden tener tal consideración las enumeradas en el art. 256 LEC. En consecuencia, debemos hablar del carácter tasado de las diligencias preliminares y, por lo tanto, "no se puede acceder a la práctica de aquellas diligencias preliminares que no se encuentren específicamente previstas en alguno de los once apartados del artículo 256.1 de la LEC"49.

\footnotetext{
${ }^{45}$ ATS (Sala Civil) 20/2002, de 11 de noviembre de 2002, f.j. 2o (ECLI: ES:TS:2002:3037A); AAP de Guadalajara (Sección 1a) 11/2007, de 2 de febrero de 2007, f.j. 1으(ECLI: ES:APGU:2007:15A); y, más recientemente, AAP Valencia (Sección 9ạ) 582/2017, de 9 de mayo de 2017, f.j. 20 (ECLI: ES:APV:2017:1804A).

${ }^{46}$ AAP de Barcelona (Sección 1a) 275/2018, de 20 de septiembre de 2018, f.j. 2ㅇ (ECLI: ES:APB:2018:5095A).

${ }^{47}$ AAP Valencia (Sección 9a) 582/2017, de 9 de mayo de 2017, f.j. 2o (ECLI: ES:APV:2017:1804A); y ATS (Sala Civil) 20/2002, de 11 de noviembre de 2002, f.j. 20 (ECLI: ES:TS:2002:3037A).

${ }^{48}$ AAP de Zamora (Sección 1a) 1/2003, de 8 de enero de 2003, f.j. 3으 (ECLI: ES:APZA:2003:2A)

49 Juzgado de lo Mercantil núm. 1 de Segovia, Auto de 7 febrero 2017, f.j. 10 (JUR 2017\39407). Sobre la sujeción de las diligencias preliminares a un numerus clausus, véase también AAP de Barcelona (Sección 1a) 275/2018, de 20 de septiembre de 2018, f.j. 2으
} 
Pese a la enumeración cerrada del art. 256.1 LEC, queremos hacer notar que a diferencia de la pre-action disclosure, las diligencias preliminares no se limitan estrictamente al ámbito documental - aunque sea el más habitual-, pues pueden solicitarse al tribunal otras actuaciones como, por ejemplo, la declaración del futuro demandado ${ }^{50}$ o la exhibición de cosas muebles o inmuebles ${ }^{51}$, en virtud de los apartados 1 y 2 , respectivamente, del citado precepto legal.

Centrándonos propiamente en los documentos, queremos señalar que la regulación inglesa - rule 31.16 (3) (c) CPR - extiende la revelación de documentos solo a los propios de la standard disclosure, lo que aparentemente puede parecer una limitación. Sin embargo, esta afirmación debe ser matizada pues cuando acudimos a la regulación de la standard disclosure nos damos cuenta de que tales documentos son todos aquellos sobre los que pueda sostenerse la pretensión, además de aquellos otros que pudieran perjudicar a la propia parte que los aportase o bien a la contraparte; e incluso los que pudieran beneficiar a la otra parte. Esto contrasta con la regulación española que solo permite la exhibición de los documentos previstos expresamente para las concretas diligencias del art. 256.1 LEC de manera que, si algún otro documento pudiera ser de utilidad para preparar el futuro proceso, pero estuviera fuera del alcance de la citada norma, no podría acordarse diligencia preliminar alguna para su exhibición.

\subsection{Requisitos}

Los arts. 258 y 256 (apartados 2 y 3) de la LEC regulan los requisitos para la adopción de las diligencias preliminares, a saber: que la diligencia sea adecuada a la finalidad que el solicitante persigue, que se fundamente la diligencia que se solicita con referencia circunstanciada al asunto objeto del juicio que se quiera preparar, y que concurran justa causa e interés legítimo.

La doctrina judicial se ha encargado de desarrollar estos requisitos. Al respecto, especialmente ilustrativo es el auto de la Audiencia Provincial de Tarragona, de 24 de septiembre de 2008, motivo por el cual traemos a colación ${ }^{52}$.

En primer lugar, quien solicite una diligencia preliminar debe estar legitimado para ello, de modo que deberá examinarse su conexión con lo que solicita ${ }^{53}$. Una similar finalidad se desprende de la rule 31.16 (3) (a) CPR al establecer que el solicitante (applicant) y el solicitado (respondent) puedan llegar a ser partes del futuro proceso judicial.

En segundo lugar, se exige que la diligencia preliminar sea adecuada a la finalidad perseguida y que la solicitud esté fundamentada. Esta exigencia ha sido desarrollada por parte de la jurisprudencia en los siguientes términos: "preparar un proceso de declaración, recabando la información necesaria o el acopio de datos y elementos precisos para decidir sobre la aptitud personal de los sujetos, activo y pasivo; de la acción que se pretenda ejercitar; sobre la existencia y circunstancias del bien sobre el cual deba versar el proceso; o sobre el alcance y extensión de las pretensiones a ejercitar (AP de Barcelona, Secc. 1. a, núm. 198/2007, de 26 de julio)”54. Aunque no se haya previsto expresamente en las CPR, esta adecuación de las diligencias preliminares al caso concreto también debe observarse en el ordenamiento jurídico inglés, tal y como han especificado, aunque de manera más genérica, los tribunales de justicia para la preaction disclosure, debiendo valorar su necesidad y las circunstancias en torno al supuesto para el que se solicita.

(ECLI: ES:APB:2018:5095A); AAP Valencia (Sección 9ạ) 582/2017, de 9 de mayo de 2017, f.j. 20 (ECLI: ES:APV:2017:1804A); y ATS (Sala Civil) 20/2002, de 11 de noviembre de 2002, f.j. 20 (ECLI: ES:TS:2002:3037A).

${ }^{50}$ AAP de Valladolid (Sección 1a) 227/2003, de 19 de septiembre de 2003, f.j. 3o (ECLI: ES:APVA:2003:215A).

${ }^{51}$ AAP de Pontevedra (Sección 1a) 102/2006, de 25 de mayo de 2006, f.j. 2으 (ECLI: ES:APPO:2006:109A).

${ }^{52}$ AAP de Tarragona (Sección 3a) 155/2008, de 24 de septiembre de 2008, f.j. 2o (JUR 2009\40603). En el mismo sentido se pronuncia el AAP de Las Palmas (Sección 4ạ) 175/2010, de 10 de junio de 2010, f.j. 60 (ECLI:ES:APGC:2010:812A)

${ }^{53}$ AAP de Barcelona (Sección 13a) 354/2005, de 13 de diciembre de 2005, f.j. 2o (JUR 2006\86061).

${ }^{54}$ AAP de Tarragona (Sección 3a) 155/2008, de 24 de septiembre de 2008, f.j. 2o (JUR 2009\40603). 
Además, para fundamentar su solicitud es indispensable "[...] que el solicitante fije, precise y determine, con claridad y concreción, cual es el objeto del juicio que se propone entablar, para qué pide la diligencia preliminar y contra quién se propone dirigir la futura demanda". En consecuencia, no se admitirán solicitudes genéricamente formuladas o basadas en vaguedades. De hecho, en parecidos términos se han pronunciado los tribunales ingleses, quienes al respecto han incidido en que, para realizar una adecuada fundamentación de la reclamación para ordenar la pre-action disclosure, deben evitarse pretensiones especulativas y poco justificadas.

En tercer lugar, la LEC requiere asimismo la concurrencia de justa causa, pero nada más indica. De nuevo ha sido la jurisprudencia quien se ha encargado de llenar de contenido esta exigencia aclarando que se trata de "[...] la justificación de la diligencia que se pide para la preparación del eventual futuro proceso", siendo necesario concretar que "[...] Todo ello supone, por su propia naturaleza meramente instrumental, preparatoria y aclaratoria, o incluso para preservar el principio de igualdad entre las partes, que: a) Solo procederán cuando haya imposibilidad de tener acceso a lo que se solicita de otro modo, esto es, que estas diligencias preliminares, como auxilio judicial a la parte que son, deben ser acordadas exclusivamente cuando no haya otro medio de preparar el ejercicio de la acción que el solicitante se propone ejercitar (Auto de la AP Barcelona núm. 73/2007, Sección 13, de 12 marzo); b) Solo procederán cuando sea necesario el conocimiento o la información que se solicita para preparar el futuro procedimiento, conocimiento o información que ha de ser esencial o relevante para tal fin; c) Que no pueden servir de instrumento para preconstituir pruebas para el futuro pleito, pues no debe confundirse diligencias preliminares con prueba anticipada, ya que son dos figuras diferentes: las diligencias preliminares tienen por objeto preparar un juicio y la prueba anticipada persigue constatar un hecho necesario para la prosperabilidad de la pretensión cuando exista un temor de que no puedan realizarse dentro del proceso". Respecto de la justa causa observamos dos puntos de conexión con la pre-action disclosure, que asimismo ha desarrollado la doctrina judicial inglesa. Por un lado, el órgano judicial deberá valorar la existencia de otras vías de obtención de los documentos cuya revelación se pretende; y, por otro lado, la relación directa de los documentos requeridos con la reclamación.

Sea como fuere, y tal y como en su momento ya indicamos para la pre-action disclosure, la última palabra para apreciar la diligencia preliminar la tendrá el órgano judicial que es quien, en última instancia, deberá valorar la necesidad y adecuación de la diligencia preliminar al caso concreto. En este sentido lo ha recordado el auto de la Audiencia Provincial de Barcelona, de 20 de septiembre de $2018^{55}$, al exigir que para acceder a la pretensión sea el juez quien "[...] aprecie si la diligencia interesada "es adecuada a la finalidad que el solicitante persigue y que en la solicitud concurren justa causa e interés legítimo»".

\subsection{Gastos y prestación de caución}

El legislador español se ha preocupado de regular quién deberá afrontar los gastos derivados de la práctica de las diligencias preliminares. En concreto, el art. 256.3 LEC establece que tales gastos serán a cargo del solicitante. Contrariamente, el legislador inglés prevé, como regla general, que sea la persona contra la que se dirige la orden judicial de pre-action disclosure (respondent) quien asuma dichos costes. En este punto, y aunque aparentemente lo parezca, entendemos que no se produce un choque frontal entre ambos sistemas jurídicos, por la distinta filosofía que se desprende de las dos instituciones procesales objeto de análisis. Como ya hemos tenido la oportunidad de indicar, en la pre-action disclosure tiene todo el sentido que los gastos repercutan en el respondent, en la medida que podría haberse evitado este trámite procesal si este hubiere atendido al pre-action protocol. En cambio, el ordenamiento jurídico español no contempla esta posibilidad siendo las diligencias preliminares la primera oportunidad que se tiene para requerir información. A pesar de ello, en nuestra opinión, el legislador español

\footnotetext{
${ }^{55}$ AAP de Barcelona (Sección 1ạ) 275/2018, de 20 de septiembre de 2018, f.j. 2o (ECLI: ES:APB:2018:5095A).
} 
debería haber sido más flexible a la hora de determinar el pago de los gastos y no imponerlos en todo caso al solicitante ${ }^{56}$.

Adicionalmente, la norma procesal española requiere también al solicitante de la diligencia preliminar el ofrecimiento de caución - sin necesidad de prestarla en el momento de presentar la solicitud (art. 256.3 LEC), y que se fijará en el auto que acuerde las diligencias preliminares (art. $258 \mathrm{LEC})^{57}$ - para hacer frente a los referidos gastos derivados de su práctica, así como a los daños y perjuicios que pudieran ocasionarse a los intervinientes en dichas diligencias. Ello no se contempla en las CPR.

\subsection{Negativa a llevar a cabo las diligencias preliminares}

Tal y como sucede en la pre-action disclosure, la práctica de las diligencias preliminares es de obligado cumplimiento por el sujeto a quien se dirijan, al ser adoptadas por un órgano judicial. Sin embargo, uno de los problemas que puede plantearse en este contexto es que, una vez dictado el auto adoptándolas, quien resulte afectado por estas no quiera colaborar en su práctica -inclusive cuando hubiere planteado oposición y se le hubiere desestimado (art. 260 LEC) - . Ante esta realidad, el legislador español ha previsto, en el art. 261 LEC, las consecuencias a la negativa de llevar a cabo las diligencias preliminares acordadas, que variarán en función del caso de que se trate.

En particular, las dos consecuencias que se prevén para las diligencias preliminares de base documental son, por un lado, la posibilidad de tener por ciertos los documentos solicitados (art. 261.4은, para los documentos contables) y, por el otro, la entrada y registro en el lugar donde se encuentren los títulos y los documentos cuya exhibición se pretende para ponerlos a disposición del solicitante en la sede del tribunal (art. 261.2은ㄷㄴ) ${ }^{58}$. A pesar de ello, la negativa a la aportación de documentos en sede de diligencias preliminares no será óbice para que quien se negó pueda aportarlos, para su valoración judicial, al posterior proceso ${ }^{59}$. Por su parte, precisamente la consecuencia que prevén las CPR, y en contraposición con el ordenamiento jurídico español, es la imposibilidad de utilizar en el posterior proceso judicial los documentos requeridos y no revelados, salvo autorización judicial.

\section{Reflexión final}

El análisis comparativo de la pre-action disclosure y las diligencias preliminares nos ha permitido revelar la importancia que tiene la colaboración entre partes antes de iniciar un proceso judicial, permitiéndose así una mejor preparación del mismo e incluso llegar a evitarlo. Este propósito se manifiesta más eficaz en el ordenamiento jurídico inglés por cuanto su legislador no solo ha optado por regularlo como un deber -en lugar de una facultad como en el caso español- sino que además lo ha extendido a un momento anterior a la preparación del futuro proceso, a través del pre-action protocol, y que mayoritariamente per se las partes cumplen. Ello tiene como consecuencia que las partes acudan solo excepcionalmente a los tribunales para solicitar una orden de pre-action disclosure. En cambio, esta fase previa a la preaction disclosure es inexistente en el ordenamiento jurídico español donde las partes no tendrán más opción que la que ofrecen las diligencias preliminares para requerir información previa a la iniciación del proceso judicial.

Como contrapartida consideramos que el ordenamiento jurídico inglés limita el derecho a la prueba al regular, como consecuencia a la negativa de aportar el documento que no se reveló o cuya inspección no se permitió, la imposibilidad de aportarlo como prueba en el

\footnotetext{
${ }^{56}$ Sobre esta cuestión, CASTRILLO (2018), pp. 133-136, propone una reforma de la LEC en el sentido de que "no [se] imponga al solicitante de las diligencias preliminares la obligación de resarcir, en todo caso, cuantos gastos, daños y perjuicios sufran quienes como requeridos intervengan en la práctica de las diligencias preliminares y aun cuando aquellos resulten probados".

${ }^{57}$ AAP de Barcelona (Sección 13a) 328/2004, de 2 de diciembre de 2004, f.j. 20 (JUR 2005\35189).

${ }^{58}$ AAP de Alicante (Sección 8a) 73/2018, de 6 de septiembre de 2018, f.j. 2o (ECLI:ES:APA:2018:272A).

${ }^{59}$ En este sentido véase CASTRILLO (2018), p. 353.
} 
posterior proceso judicial, haciendo decaer de entrada su posible valor probatorio. Más permisivo en este punto es el ordenamiento jurídico español que, ante la negativa de exhibición documental en sede de diligencias preliminares, igualmente permite su posterior aportación como prueba al proceso para que el órgano judicial pueda, según las reglas correspondientes, decidir sobre su admisión, preservándose así el derecho fundamental a la prueba del art. 24.2 de la Constitución Española ${ }^{60}$.

\section{BIBLIOGRAFÍA CITADA}

Ashfield, evan; Browne, Julie; Jolly, Peter; Keane, Adrian; laurenti, lisa; lidbetter, Andrew; OWens, Alan; Petroldi, Anna; Rose, William; Scott, Charles; Weiniger, Matthew (2015): Blackstone's Civil Practice. The commentary 2015, 4a edición (Oxford, Editorial Oxford University Press).

BLACKIE, KIM Y BATES, JOHN (2014): Civil Litigation In Practice (Newcastle, Editorial Northumbria Law Press).

CASTRILLO SANTAMARÍA, REBECA (2018): La preparación del proceso civil: las diligencias preliminares (Barcelona, Editorial J. M… Bosch).

Cerrato Guri, Elisabet (2015): "Los medios de prueba en las civil procedure rules inglesas", en: Justicia, Revista de Derecho Procesal (№ 1), pp. 309-333.

CoRBAL HERnÁNDEZ, Jesús EUGENIO (2014): “Diligencias preliminares (arts. 256 a 263)”, en Corbal, Jesús; Izquierdo, Pablo y Picó, Joan (Dirs.), Práctica procesal civil, Brocá-Majada-Corbal, 23a edición (Barcelona, Editorial Bosch), tomo IV, pp. 3483-3602.

CUNNINGHAM-HILL, SUSAN Y ELDER, KAREN (2013): Civil Litigation Handbook (Oxford, Editorial Oxford University Press).

De Prada Rodríguez, Mercedes y Muñoz Rojo, Roberto (2014): El proceso civil inglés (Granada, Editorial Comares).

DI MAMBRo, DAVID (2019): The Civil Court Practice 2019 (London, Editorial LexisNexis), volumen 1.

Hollander, CHARLES (2015): Documentary Evidence, 12a edición (London, Sweet \& Maxwell).

ILLER, MARTIN (2006): Civil Evidence: The Essential Guide (London, Editorial Thomson Sweet \& Maxwell).

Matthews, Paul y Malek, Hodge M. (2017): Disclosure (London, Sweet\&Maxwell - Thomson Routers).

PeÑA, CRISTÓBAL (2017): "Traduciendo el discovery al civil law chileno: Su aporte a los procesos de reforma procesal civil", en: lus et Praxis (Vol. 23, № 2), pp. 79-120.

PICó, JOAN (1996): El derecho a la prueba en el proceso civil (Barcelona, Editorial J. Mạ. Bosch).

RICHARD, MANUEL (2019): “¿Diligencias preliminares para preparar el proceso o como medio para obtener judicialmente datos protegidos?", en: Diario La Ley (№ 9533), pp. 1-12.

SMITH, GRAEME (2015): Civil Court Service (Bristol, Editorial Jordan Publishing).

ZUCKERMAN, AdRIAN (2013): Zuckerman on Civil Procedure. Principles of Practice (London, Sweet \& Maxwell - Thomson Reuters).

\footnotetext{
${ }^{60}$ Sobre la protección constitucional del derecho a la prueba en el proceso civil véase ampliamente a PICÓ (1996).
} 


\section{JURISPRUDENCIA CITADA}

BERMUDA INTERNATIONAL SECURITIES LTD VS KPMG (A FIRM) [2001] EWCA CIV 269, (2001) 145 SOL JO LB 70 (United Kingdom).

BLACK VS. SUMITOMO CORPORATION [2001] EWCA CIV 1819, [2002] (United Kingdom). HERBERT BLACK AND OTHERS VS. SUMITOMO CORP [2002] 1 WLR 1562 (United Kingdom).

arsenal Football Club VS. Elite SPORTS Distribution [2002] EWHC 3057 (CH), LIGHTMAN J. (United Kingdom).

AUTO DEL TS (SALA CIVIL) 20/2002, de 11 de noviembre de 2002 [ECLI: ES:TS:2002:3037A] (España). MORESFIELD VS. BANNERS [2003] EWHC 1602 (CH) (United Kingdom).

SNOWSTAR SHIPPING Co LTD VS. GRAIG SHIPPING PLC [2003] EWHC 1367 (COMM) (United Kingdom). JAY VS. WILDER COE [2003] EWHC 1786 (QB) TUGENHART H. (United Kingdom).

Auto DE LA AP DE ZAMORA (SECCIÓN 1ạ) 1/2003, DE 8 DE ENERO DE 2003 [ECLI: ES:APZA:2003:2A] (España).

Auto de la AP de Valladolid (SeCción 1a) 227/2003, de 19 de SePtiembre de 2003 [ECLI: ES:APVA:2003:215A] (España).

XL LONDON MARKET LTD VS. ZENITH SYNDICATE MANAGEMENT LTD [2004] EWHC 1182 (COMM) (United Kingdom).

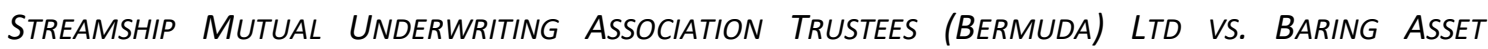
MANAGEMENT LTD [2004] EWHC 202 (COMM), [2004] ALL ER (D) 272 (FEB), AIKENS J. (United Kingdom).

Marshall (EXeCUtors of Marshall) vs. Alliotts (A FIRM) [2004] EWHC 1964 (QB), [2005] United Kingdom).

Auto de LA AP DE BARCELONA (SECCIÓN 13a) 328/2004, DE 2 DE DICIEMBRE DE 2004 [JUR 2005\35189] (España).

First Goulf Bank VS. Wachovia Bank National AsSOCIATION [2005] EWHC 2827 (COMm) United Kingdom).

NoRTHUMBRIAN WATER LTD VS. BRITISH TELECOMMUNICATIONS PLC [2005] EWHC 2408 (TCC) (United Kingdom).

BRIGGS \& FORRESTER ELECTRICAL LTD VS. GOVERNORS OF SOUTHFIELD SCHOOL [2005] EWHC 1734 (TCC) United Kingdom).

Auto de LA AP de BARCELona (SeCCIÓN 13a) 354/2005, DE 13 de diCIEMBRE DE 2005 [JUR 2006\86061] (ESPAÑA).

HANDS VS. MorRISON CONSTRUCtION SERVICES LTD [2006] EWHC 2018 (СH) (United Kingdom).

BSW LTD VS. BALLTEC LTD [2006] EWHC 822 (CH) United Kingdom).

Auto de la AP de PonteVedra (Sección 1a) 102/2006, DE 25 de mayo de 2006 [ECLI: ES:APPO:2006:109A] (España).

Auto de la AP de Guadalajara (SecCión 1a) 11/2007, de 2 de febrero de 2007 [ECLI: ES:APGU:2007:15A] (España).

HAYS SPECIALIST RECRUITMENT (HoldINGS) LTD VS. IONS [2008] EWHC 745 (CH) United Kingdom). 
GWELHAYL LTD VS. MIDAS CONSTRUCTION LTD [2008] EWHC 2316 (TCC) United Kingdom).

Auto de LA AP de TARRAGONA (SECCIÓN 3a) 155/2008, DE 24 DE SEPTIEMBRE DE 2008 [JUR 2009\40603] (España).

EDO CORPoration Vs. UltRAELECTRONICS LTD [2009] EWHC 682 (CH), [2009] Bus LR 1306 United Kingdom).

GAS MARKETING LTD VS. CENTRICA StORAGES LTD [2009] EWHC 732 (CH) United Kingdom).

ANGLO IRISH BANK CORPORATION PLC VS. WEST LB AG [2009] EWHC 207 (COMM), LTL25/2/2009 (United Kingdom).

KNEALE VS. BARCLAYS BANK (T/A BARKLAYCARD) [2010] EWHC 1900 (COMM) United Kingdom).

PINEWAY LTD. VS. LONDON MINING CO LTD [2010] EWHC 1143 (COMM) (United Kingdom).

Auto de LA AP DE LAS PALMAS (SecCión 4a) 175/2010, DE 10 DE JUNIO DE 2010 [ECLI:ES:APGC:2010:812A] (España).

SMith VS. SeCRetary of State for the ENVIRONMENT AND Climate Change [2013] EWCA Civ 1585, [2014] 1 WLR 2283, UNDERHILL L (United Kingdom).

JET AIRWAYS (INDIA) VS BARLOWORLD HANDLING LTD [2014] EWCA CIV 1311 (United Kingdom).

Juzgado de lo Mercantil núm. 1 de Segovia, Auto de 7 febrero 2017 [JUR 2017\39407] (España).

Auto de LA AP VALENCIA (SECCIÓN 9a) 582/2017, DE 9 DE MAYO DE 2017 [ECLI: ES:APV:2017:1804A] (España).

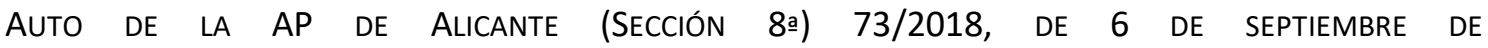
2018[ECLI:ES:APA:2018:272A] (España).

Auto de la AP de BARCELONA (SECCIÓN 1a) 275/2018, DE 20 DE SEPTIEMBRE de 2018 [ECLI: ES:APB:2018:5095A] (España).

\section{NORMAS JURÍDICAS CITADAS}

CONSTITUCIÓn ESPAÑOLA. Boletín Oficial del Estado, núm. 311, de 29 de diciembre de 1978.

LEY № 1, DE ENJUICIAMIENTO CIVIL. Boletín Oficial del Estado, núm. 7, de 8 de enero de 2000.

Rule № 3132, The CiVIL Procedure Rules. UK Statutory Instruments, de 10 de diciembre de 1998.

Ley 1552, Código de Procedimiento CiVIL. Diario Oficial, 30 de agosto de 1902. 